\title{
Energy efficiency of temperature distribution in electromagnetic welding of rolling stock parts
}

\author{
Andrew Lyudagovsky ${ }^{1}$, Alexey Loktev ${ }^{1}$, Vadim Korolev ${ }^{1}$, Irina Shishkina ${ }^{1,{ }^{*}}$, Diana Alexan- \\ drova $^{1}$, Pavel Geluh ${ }^{1}$, and Daniil Loktev ${ }^{2}$ \\ ${ }^{1}$ Russian open Academy of transport of the Russian University of transport, Chasovaya str. 22/2, Mos- \\ cow, 125190, Russia \\ ${ }^{2}$ Moscow State University of Civil Engineering (MGSU), Yaroslavskoye Shosse, 26, Moscow, 129337, \\ Russia
}

\begin{abstract}
When modeling temperature fields in details of different geometry on the basis of the obtained calculations, the graphs of temperature dependence on the power of the source and the thickness of the deposited layer, non-contact measurement of temperatures in the working area and the subsequent calculation of temperature fields allow describing the thermal processes in the electromagnetic surfacing in the first approximation. The determination of temperatures should be carried out taking into account the accumulation of heat by an infinite cylinder with a constant source evenly distributed over its lateral surface.
\end{abstract}

\section{Research technique}

This work is devoted to the modeling of temperature fields in details of different geometry. If the sizes of the restored parts are not large, and the heat sink is not intense enough, then we can consider the accumulation of heat, leading to an increase in the temperature of the workpiece in the recovery process. To do this, imagine that the fast-moving source moves along the surface of a solid cylinder along the helix of a small step $2 \mathrm{~b} 0$ and makes several turns (Fig.1).

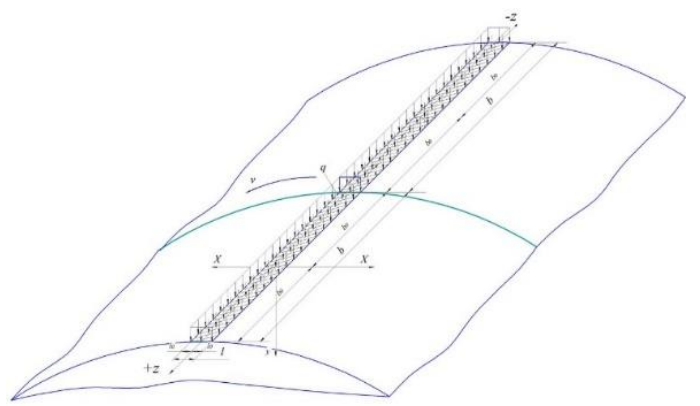

Fig. 1. Scheme of temperature fields from the strip fast-moving heat source

\footnotetext{
* Corresponding author: shishkinaira@inbox.ru
} 
Then the temperature of an arbitrary point on the surface by radius will be expressed as the sum of temperatures from instantaneous annular sources, which can be described by the following relation:

$$
T_{\mathrm{K}}=\frac{2 q}{c \rho(4 \pi \lambda)^{3 / 2}} \cdot e^{-\frac{v \tau}{2 \lambda}} \int_{0}^{\tau_{n}} e^{-\frac{v^{2} \tau}{4 \lambda}-\frac{R_{\mathrm{K}}^{2}}{4 \lambda \tau}} \cdot \frac{d \tau}{\tau^{3 / 2}}
$$

where $c$ - mass heat capacity, $\mathrm{J} /\left(\mathrm{kg} \quad{ }^{\circ} \mathrm{C}\right) ; \rho$ - density of matter, $\mathrm{kg} / \mathrm{m}^{3}, c \rho$-volume heat capacity, $\mathrm{J} /\left(\mathrm{cm}^{3}{ }^{\circ} \mathrm{C}\right) ; \lambda$ - coefficient of thermal diffusivity, $\mathrm{cm}^{2} / \mathrm{c} ; v$ - speed of movement of the source, $\mathrm{cm} / \mathrm{c} ; \tau_{n}$ - time of movement of the source, $\mathrm{c} ; R_{\kappa}$ - instantaneous coordinates of the considered point, $\mathrm{cm} ; q$ - point source of heat of constant power, $\mathrm{J} / \mathrm{c}$;

$$
q=\eta \cdot V \cdot I
$$

where $\eta$ - the efficiency of the heat source; $V$ - voltage, V; $I$ - current strength, A.

When determining the optimum temperature of the metal coating, after the termination of the surfacing process, it is necessary to proceed from the fact that the processing of a solid cylinder along the helix with a small step should be considered as heating by a fast-moving point source moving along its surface [1-3].

This assumption means that the heat is distributed only in a line bounded by two nonheat-permeable planes and passing through the axis of the cylinder and the outer surface from which the heat transfer occurs. It is taken into account that the angle of rise of the helix when surfacing with a small step is relatively small, the distribution of heat in this case can be equated to the case of its propagation from an instantaneous annular source with linear energy on the surface of the cylinder. Then the temperature of an arbitrary point on the surface with radius $R$ will be expressed as a sum of temperatures from instantaneous annular sources, which can be described by the ratio:

$$
T_{\mathrm{K}}=\frac{2 q_{\mathrm{K}}}{v_{n} c \rho R} \cdot \sum_{n=1}^{N} \Phi_{\Pi}(r, \tau) \frac{e^{\frac{-x_{n}^{2}-B \tau_{n}}{4 \omega \tau}}}{\sqrt{4 \pi \omega \tau_{n}}}
$$

where $q_{k} / v_{n}$ - heat input from the instant of the annular source; $c \rho$ - the specific heat of the material $\mathrm{J} / \mathrm{cm}^{3}$ grad; $r$ - the current radius, $\mathrm{cm} ; R$ - the estimated radius of the part after welding, $\mathrm{cm} ; \omega$ - thermal diffusivity, $\mathrm{cm}^{2} / \mathrm{c} ; \tau_{n}$ - the time elapsed from the moment of crossing the heat source of the plane $I-I$ in respective passageways (Fig.2), $z_{n}$ - the distance from the heat source to the point in the plane under consideration at different passes, i.e. at $z_{1}, z_{2}$, $\mathrm{z}_{3} \ldots . ., \mathrm{cm} ; B$ - temperature coefficient, $1^{\circ} / \mathrm{c} ; \Phi_{\mathrm{n}}(r, \tau)$ - function describing the process of heat equalization in a thin round disk without heat transfer at instantaneous heat release along the ring on its outer surface.

$$
\Phi_{\Pi}(r, \tau)=\sum_{k-1}^{\infty} \exp \left(-\frac{\mu^{2} k \omega \tau}{R^{2}}\right) J_{0}\left(\frac{\mu_{k}}{R}\right) / J_{0}\left(\mu_{k}\right)
$$

where $J_{0}, J_{1}$ - Bessel functions of the first kind of the first order, $\mu_{k}$ the roots of the equation $J_{1}\left(\mu_{k}\right)=0,\left(\mu_{1}=0 ; \mu_{2}=3.83 ; \mu_{3}=7.02\right)$ 


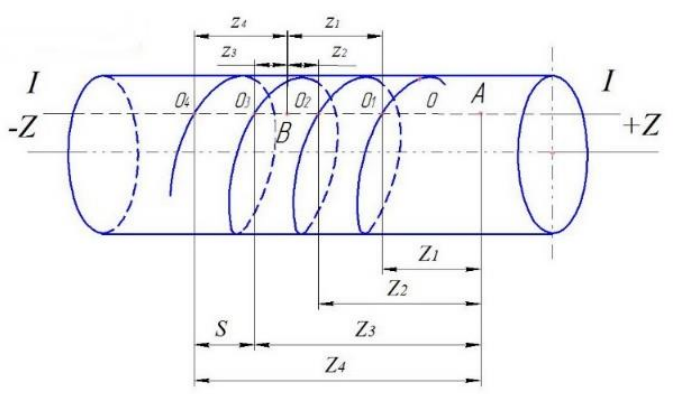

Fig. 2. Scheme of motion and location of heat sources when heating a solid cylinder on a small step helical line

The values $\Phi_{\Pi}(r, \tau)$ can also be determined by the nomogram given in [1] depending on $\frac{r}{R}$ и $\tau=\frac{\omega t}{R^{2}}$.

In the case of a steady-state process, when the number of passes $N$ is large, the summation of temperatures according to the formula (3) should be carried out to the value $n=N_{0}$, while it $\Phi_{\Pi}(r, \tau)$ differs from one by 3-5\%. In this case, the temperature $T_{N_{0}}$, will be obtained which is determined by the formula:

$$
T_{N_{0}}=\frac{2 q_{k}}{v R v k} \sum_{n=1}^{N} \Phi_{\Pi}(r, \tau)
$$

The remainder of the sum of equation (3) at $n>N_{0}$, when $\Phi_{\Pi}(r, \tau) \cong 1$ is produced using the integral:

$$
T_{N-N_{0}}=\frac{q e^{-\frac{v_{Z}^{2}}{2 \omega}}}{c \rho F \sqrt{4 \pi \omega}} \int_{\left(\tau_{N_{0}}+\frac{\tau_{S}}{2}\right)}^{\infty} e^{-\left(\frac{v_{Z}^{2}}{4 \omega}+B\right) \tau-\frac{x_{0}^{2}}{4 \omega \tau}} \cdot \frac{d \tau}{\sqrt{\tau}},
$$

where $\tau_{N_{0}}$ - the time since the intersection of the plane $I-I$, when passing $n=N_{0}$

$\tau_{s}-$ the time spent on a single pass with a step s, (Fig. 2);

$x_{0}-$ coordinate of the point $A$ or $b$ (Fig. 2) in relation to the moving heat source $Q$;

$v_{z}$ - component of the velocity of the heat source along the axis of the cylinder $z$.

Integral (6) describes the process of temperature equalization from a flat heat source, i.e.:

$$
T_{N-N_{0}}=T_{\text {пр }}\left(1-\psi_{1}\right),
$$

where $\psi$ is determined by the nomogram [1], а $T_{\text {пр }}$ :

$$
T_{\text {пр }}=\frac{q}{c \rho \pi R^{2} \sqrt{1+\frac{4 B \omega}{v^{2}}}} e^{-\frac{v_{Z}}{2 \omega}-\frac{v|z|}{2 \omega} \sqrt{1+\frac{4 B \omega}{v^{2}}}},
$$

Finally get:

$$
T=T_{N_{0}}+T_{N-N_{0}}=T_{N_{0}}+T_{\text {пр }}\left(1-\psi_{1}\right),
$$

On the basis of the obtained formulas, to determine the theoretical distribution of the temperature field in the surfacing of solid cylindrical parts in the Mathcad system, we take the following values of thermal coefficients taking into account the average heating temperature of 
$600{ }^{\circ} \mathrm{C}: c \rho=5 \mathrm{~J} / \mathrm{cm}^{3} \operatorname{grad} ; \omega=0.07 \mathrm{~cm}^{2} / \mathrm{c} ; \lambda=0.3 \mathrm{~J} / \mathrm{cm}^{2} \mathrm{c} \operatorname{grad} ; \alpha=6 \cdot 10^{-3} \mathrm{~J} / \mathrm{cm}^{3} \mathrm{c} \operatorname{grad} ; \eta$ $=0.7$.

The calculation of the power source depends on the following values of the discharge current $\mathrm{I}=120,130,140,150 \mathrm{~A}$, voltage $22 \mathrm{~V}$. The thickness of the cladding is taken 100, 200, 300,400 microns. The calculations are made for the temperature of the point located at a depth of $3.5 \mathrm{~mm}$ from the surface, taking into account the deposited layer and at a distance of $30 \mathrm{~mm}$ behind the heat source for a solid cylinder with a diameter of $6.5 \mathrm{~cm}$, taking into account more than 3 welding passes.

\section{Results}

On the basis of the obtained calculations, the graphs of temperature dependence on the power of the source and the thickness of the deposited layer are constructed (Fig.3.4).

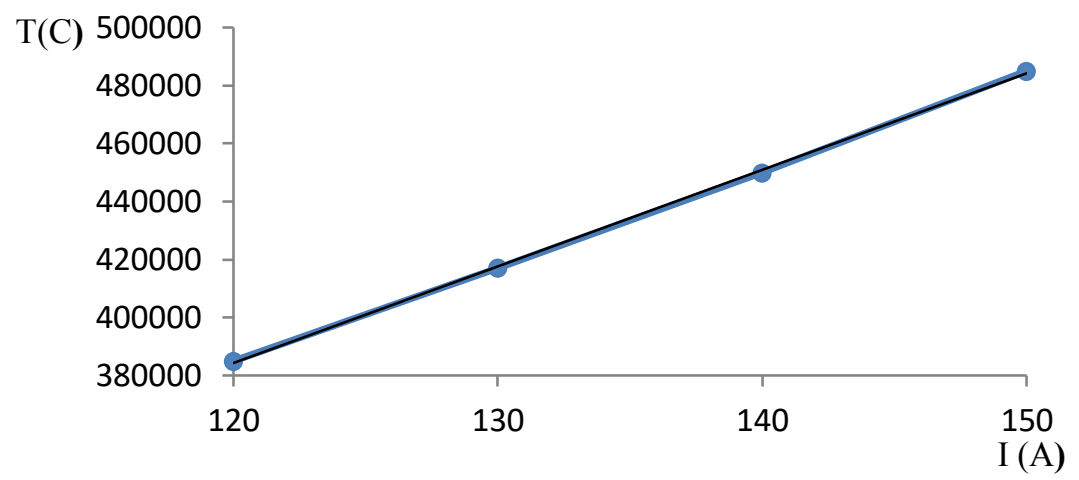

Fig. 3. The graph of temperature change in the invested point from the change of power source by increasing the current with a coating thickness of 400 microns

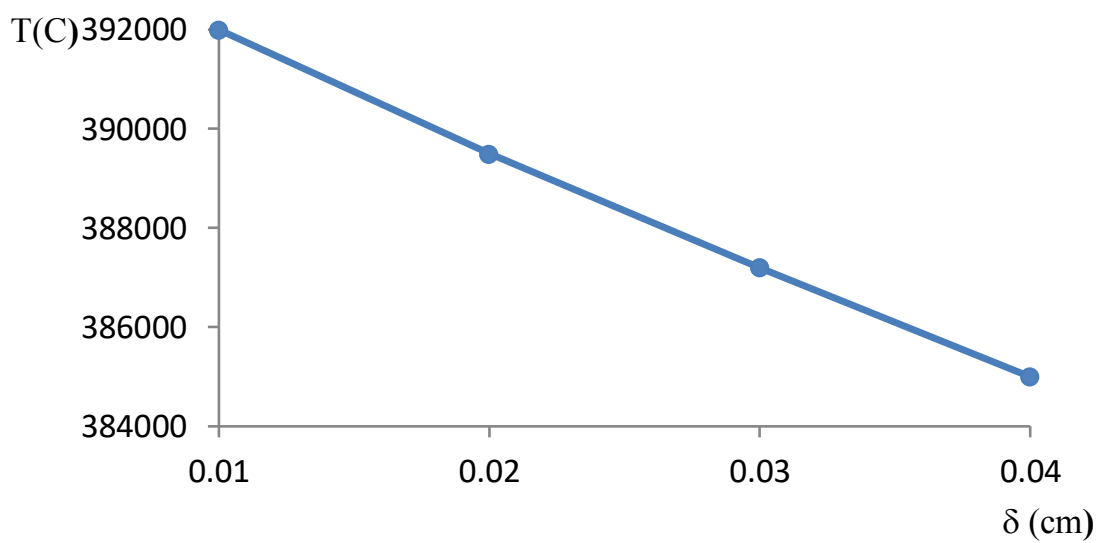

Fig. 4. The graph of temperature change in the test point from the coating thickness change at a constant power source current of $120 \mathrm{~A}$

As can be seen from figure 3, the temperature at the test point increases proportionally with increasing power source and at the same time decreases proportionally depending on the thickness of the coating at a constant power source of heat (Fig.4).

With a large number of passes $\Phi_{\Pi}(r, \tau)>1$ means that the cylinder is almost completely warmed up in thickness, thereby violating the accepted scheme of the process and the calculation of temperature fields according to the formulas (7-9) is impractical. 
In this case, the determination of temperatures should be carried out taking into account the accumulation of heat by an infinite cylinder with a constant source evenly distributed over its side surface. Then, provided that the heat exchange with the environment is established and the temperatures on the side surface are aligned, it is possible to consider a nonstationary one-dimensional problem with boundary conditions of the first kind, in which the heating source is represented by a permanent and annular. The differential equation of thermal conductivity in this case will be presented:

$$
\frac{\partial T}{\partial \tau}=\omega\left(\frac{\partial^{2} T}{\partial r^{2}}+\frac{1 \partial T}{r \partial r}\right)
$$

where $\tau>0,0<r<R$ initial conditions $T(r, 0)=T_{0}=$ const and boundary conditions $T(r, 0)=T_{c}=$ const, $\frac{\partial T(0, \tau)}{\partial r}=0, T(0, \tau) \neq \infty$.

The latter condition means that the temperature in the center of the ring throughout the heat exchange process must be finite.

The solution of the equation (10) is similar to the solution of the temperature equalization problem (4):

$$
\frac{T_{c}-T}{T_{c}-T_{0}}=\sum_{k-1}^{\infty} 2 \exp \left(-\frac{\mu_{k}^{2} \omega \tau}{R^{2}}\right) J_{0}\left(\frac{\mu_{k}^{2} r}{r}\right) /\left[\mu_{n} J_{1}\left(\mu_{n}\right)\right]
$$

where $\mu_{n}=(2 n-1) \pi / 2$.

In cases where the end surfaces are restored and the length of the cylindrical surface is small, the heat accumulation process should be considered with a steady heat exchange for a limited cylinder with constant temperatures on its side and end surfaces.

\section{Conclusions}

1. On the basis of the obtained calculations, the graphs of temperature dependence on the power of the source and the thickness of the deposited layer are constructed.

2. Non-contact measurement of temperatures in the working area and the subsequent calculation of temperature fields allow describing thermal processes at the electromagnetic surfacing in the first approximation.

3. Determination of temperatures should be carried out taking into account the accumulation of heat by an infinite cylinder with a constant source evenly distributed over its side surface.

\section{References}

1. A. A. Loktev, S. A Matasov, T-comm. Telecommunications and transport, 7(3), 28-31 (2013)

2. A. A. Loktev, Mechanics of composite materials and structures, 13(3), 170-178 (2007)

3. A. A. Loktev, Applied mathematics and mechanics, 72(4), 652-658 (2008)

4. A. A. Loktev, Mechanics of composite materials and structures, 11(4), 478-492 (2005)

5. A. A. Loktev, Letters to the Journal of technical physics, 33(16), $72-77$ (2007)

6. Y. B. Barkovskiy, M. N. Gorokhova, V. N. Retyunskikh, Technology of metals, 12, 3436 (2007)

7. A. A. Loktev, D. A. Loktev, Technical Physics Letters, 34(11), 960-963 (2008)

8. D. N. Byshov, D. G. Churilov, A. A. Gorokhov, Bulletin of Ryazan state agrotechnological University. P. A. Kostychev, 3(15), 66-68 (2012) 